\title{
Large Gastritis Cystica Polyposa Treated with EMR Polypectomy in Patient without History of Gastric Surgery
}

\section{Seyed Reza Fatemi ${ }^{1 *}$, Behzad Hatami ${ }^{1}$, Naghmeh Salarieh ${ }^{1}$, Azam Farahani $^{1}$, Seyed Ali Fatemi ${ }^{2}$, Sam Ali Zadeh ${ }^{2}$ and Mahsa Molaei ${ }^{3}$}

${ }^{1}$ Gastroenterology and Liver Diseases Research Center, Research Institute for Gastroenterology and Liver Diseases, Shahid Beheshti University of Medical Sciences, Tehran, Iran

${ }^{2}$ Life Science, Biology, Student, University of Toronto, Canada ${ }^{3}$ Pathology Department, Taleghani Hospital, Shahid Beheshti University of Medical Science, Tehran, Iran

*Corresponding Author: Seyed Reza Fatemi, Gastroenterology and Liver Diseases Research Center, Research Institute for Gastroenterology and Liver Diseases, Shahid Beheshti University of Medical Sciences, Tehran, Iran.
Received: June 11, 2021

Published: June 26, 2021

(c) All rights are reserved by Seyed Reza

Fatemi., et al.

\section{Abstract}

Gastritis cystica polyposa (GCP) is a rare inflammatory disease of the gastric remnant that usually develops after partial gastrectomy. It is defined by the presence of polyps on anastomotic gastric junction mucosa, at histopathological examination, by the presence of mucosal and submucosal cysts with foveolar hyperplasia. The disease is characterized by the frequency of gastro-intestinal bleeding and possibility of carcinomatous association.

The endoscopic examination of a 51-year-old female without history of gastric surgery revealed a protruding lesion in the greater curvature of the middle third area of stomach. The lesion was $25 \mathrm{~mm}$ in size. Polypectomy was performed with snare base method and submucosal stalk injection, with endoscopic impression of hyperplastic polyp, without any complications.

Histopathological findings were consistent within gastritis cystic polyposa (GCP).

Keywords: Gastric Polyp; Gastritis Cystica Polyposa; Endoscopic Polypectomy

\section{Introduction}

Gastritis cystica polyposa is a unique lesion, found on occasion at the stoma of a gastrojejunal anastomosis, long time after distal partial gastrectomy. The pathological criteria were first described by Nicolai and Mueller [1] in 1965 and the term "gastritis cystica polyposa (GCP)" was proposed by Littler and Gleibermann in 1972 [2]. Later, in 1979, Koga., et al. named the same lesion stomal polypoid hypertrophic gastritis (SPHG), and both terminologies are equally prevalent today [3].
GHPs are usually asymptomatic and therefore they are often found incidentally during upper endoscopy that is performed for various reasons, GCP can present as an abdominal pain, nausea, anorexia, gastrointestinal bleeding, acid reflux and gastric outlet obstruction [24]. Itte., et al. [26] reported a case of a 50-year-old man with massive gastrointestinal hemorrhage due to GCP.

GCPs usually appear in upper endoscopy as a giant gastric fold, a submucosal tumor, or isolated polyps. 
Pathological presentation is a cystic dilation of the gastric glands in the submucosa [23]. However, little basic studies have been done, and the mechanism of such potential carcinogenesis is not yet clear. Gastritis cystica polyposa (GCP) is an uncommon lesion that develops in patients who have undergone gastroenterostomy with or without gastric resection and typically occurs at anastomosis site [18-21]. GCP is rarely found in an unoperated stomach [19-22] which usually occurs in the fundus part of stomach [23].

\section{Case Report}

A 51-year-old woman was admitted because of abdominal discomfort. 13 years prior to the current complaints, she had experienced polypectomy of colon polyps. She had recently complained of epigastric pain and vomiting.

Physical examination revealed no significant findings. Hemoglobin was $9.5 \mathrm{~g} / 100 \mathrm{ml}$, although two stool specimens were negative for occult blood.

No specific familial or past medical history was identified. Routine hematological examination and biochemical tests were within normal limits, except mild to moderate anemia.

Endoscopic examination of the upper digestive tract revealed a pedunculated polyp, about $25 \mathrm{~mm}$ in diameter in greater curvature of the middle third area of the stomach (Figure 1). We had to make a differential diagnosis between a large hyperplastic polyp and a submucosal tumor covered with normal gastric mucosa.

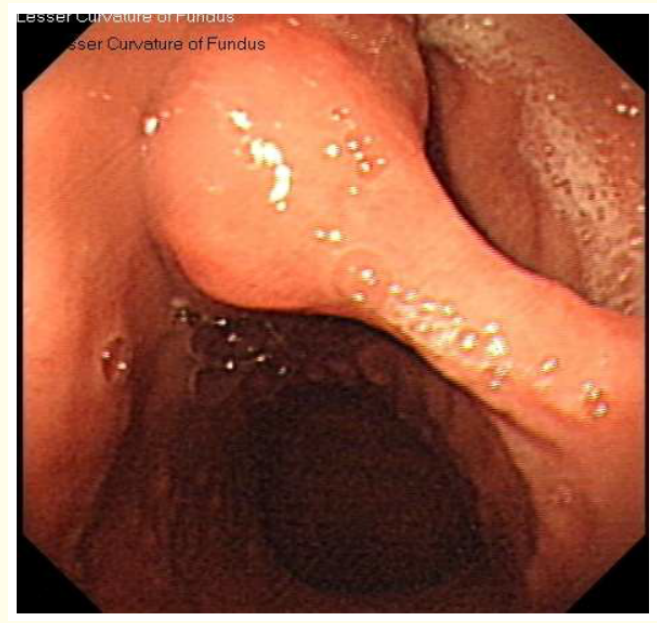

Figure 1: Endoscopy revealed a large pedunculated polyp in midportion of greater curvature.
Endoscopic ultrasonography (EUS) with a probe of $12 \mathrm{MHz}$ frequency using the water filling method revealed a polypoid heteroechoic mass with cystic part and $20 \times 22 \mathrm{~mm}$ diameter in posterior wall of gastric body that originated from mucosa and lamina propria (Figure 2). Polypectomy was performed without any complications.
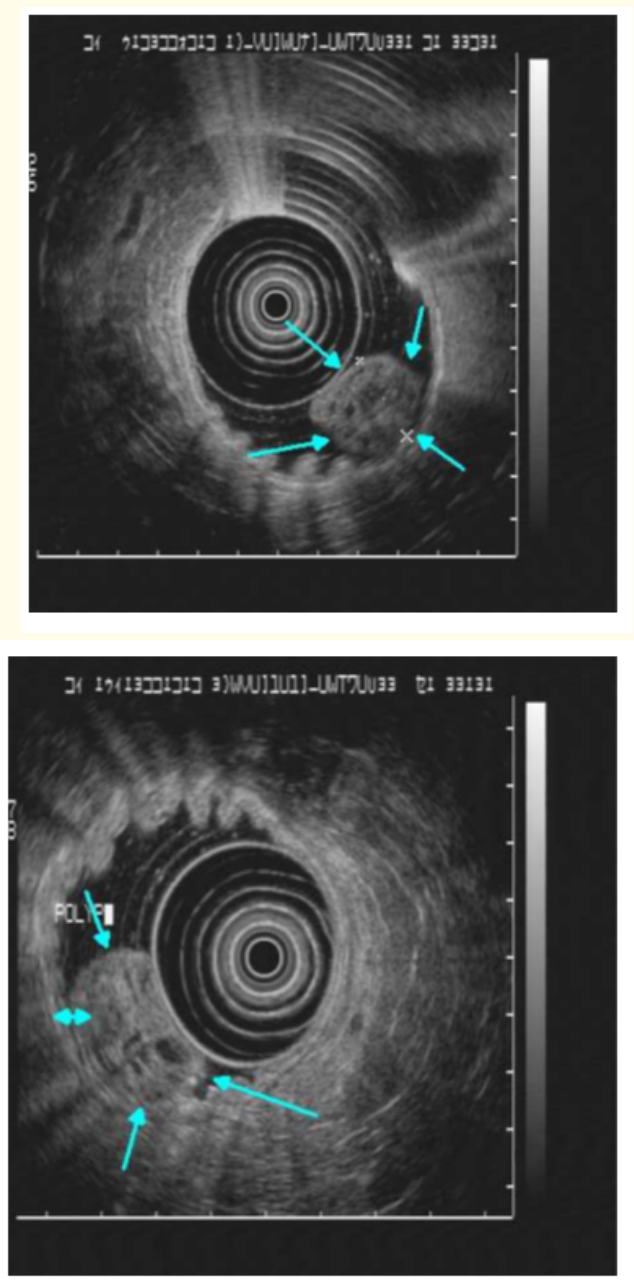

Figure 2: Endoscopic ultrasonography showed a polypoid heteroechoic mass with cystic area in posterior wall of gastric body, in mucosa and lamina propria.

Histological examination of the protruding lesion revealed the resected polypoid lesion measured $2.5 \times 1.5 \times 1.5 \mathrm{~cm}$ in size with a short stalk. Cut sections disclosed a number of small mucin-filled cysts with a background of pinkish stroma. On microscopic exami- 
nation, surface of polyp showed antral type mucosa with focal goblet cell metaplasia and moderate chronic inflammation.

In depth of polyp, some misplaced cystic glands were entrapped in dense disorganized bundles of smooth muscle of muscularis mucosa. These glands covered by flat or glandular epithelium and surrounded by a rim of lamina propria. Dysplasia or malignancy was not identified.

These findings were consistent with gastritis cystic polyposa (Figure $3 \mathrm{a}$ and $3 \mathrm{~b}$ ).

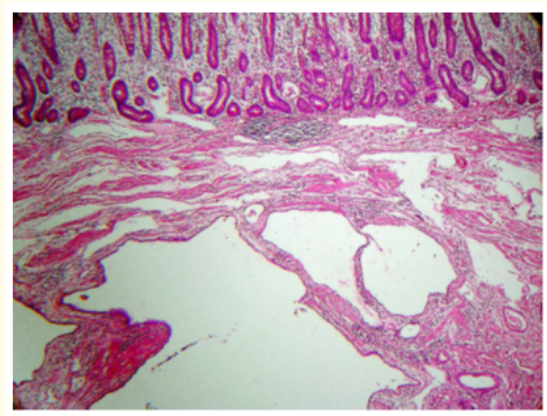

a

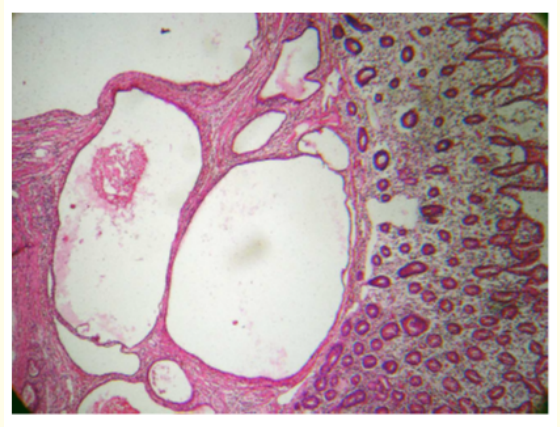

b

Figure 3a and 3b: Histopathological examination of the polyp revealed numerous misplaced cystic glands lined by flat gastric epithelium, entrapped in dense disorganized bundles of smooth muscle (Hematoxylin \& Eosin× 100).

\section{Discussion and Conclusion}

Gastritis cystica polyposa (GCP) is a unique type of chronic gastritis represented by a sessile elevated lesion along suture line, occurring a long time after Billroth-II surgery or simple gastrojejunal anastomosis. Recently, this lesion has begun to attract the interest of researchers in respect to its relationship to carcinogenesis.
Many of the GCP lesions reported in the past were found in association with early or small cancerous lesions which had developed just inside or adjacent to the GCP mucosa [4-7]. It is commonly accepted that the reason GCPs develop almost exclusively at the stoma, is that the pathogenesis of this lesion is derived from the duodenogastric reflux (DGR) [8] so that most reported GCPs are found in remnant stomach that had Billroth-II surgery.

In Western countries, it is widely accepted that a remnant stomach after partial gastrectomy for peptic ulcer disease has an increased risk of developing carcinoma, with a latency of more than 20 years [9-14], the risk being three to fivefold higher than matched population group [10]. It has also been widely recognized that treatments with Billroth-II or simple gastrojejunostomy lead to higher risks of the development of stump carcinomas than those with Billroth-I or other non-reflux types of anastomosis $[10,15]$.

GCP is typically found at sites of previous surgeries, it was suggested that mucosal injury caused by surgery or the suture technique itself promotes chronic mucosal ischemia and inflammation, prolapse and herniation glands into the submucosa with subsequent atrophic gastritis, intestinal metaplasia and cystic dilation of gastric glands [24].

Wang., et al. [17], reported a case of GCP recurrence within 6 months after surgical resection. therefore, long-term follow up is needed.

In study by Congyang Li., et al. [23] that evaluated clinical and pathologic study of seven cases with GCP, all cases showed dilated tubular glands, mainly located in the submucosa, among the muscularis mucosa and lamina propria. In our study the pathological finding were found to be similar to this article.

Xiung Fe Yu., et al. [25] reported a case of a $2.5 \mathrm{~cm}$ GCP in a 43year old woman that underwent a distal gastric resection (Billroth $1)$.

Bechade D., et al. [26] reported a case of GCP in a patient with no history of gastric surgery that presented as giant gastric fold.

Eventually in our case the patient had no history of previous surgery and GCP was successfully resected during polypectomy procedure. 


\section{Bibliography}

1. Nicolai N and Mueller D. "Des klinische und pathologischanatomische Bild der Gastritis Cystica”. Bruns Beiträge zur klinischen Chirurgie 210 (1965): 367-378.

2. Littler ER and Gleibermann E. "Gastritis cystica polyposa (gastric mucosal prolapse at gastroenterostomy Site, with cystic and infiltrative epithelial hyperplasia)". Cancer 29 (1972): 205209.

3. Koga S., et al. "Stomal polypoid hypertrophic gastritis. A polypoid lesion at Gastroenterostomy site". Cancer 43 (1979): 647657.

4. Qizilbash AH. "Gastritis cystica and carcinoma arising at old gastrojejunostomy stoma”. Canadian Medical Association Journal 112 (1977): 14323.

5. Bogomolets WV., et al. "Pathological features and mucin histochemistry of primary gastric stump carcinoma associated with gastritis cystica polyposa. A study of six cases". The American Journal of Surgical Pathology 9 (1985): 401-410.

6. Ishikawa H., et al. "A case of early gastric stump Carcinoma occurring in pedunculated gastritis cystica polyposa (in Japanese)". Gastroenterology and Endoscopy 29 (1987): 2247-2252.

7. Markowski AR., et al. "Pathophysiological and clinical aspects of gastric hyperplastic polyps". World Journal of Gastroenterology 22.40 (2016): 8883-8891.

8. Jablokow VR., et al. "Gastric stomal polypoid hyperplasia: report of four cases”. Journal of Surgical Oncology 19 (1983): 106108.

9. Arnthorsson G., et al. "Gastric cancer after gastrectomy". International Journal of Cancer 42 (1988): 365-367.

10. Caygill CPJ., et al. "Mortality from gastric cancer following gastric surgery for peptic ulcer". Lancet (1986): 929-931.

11. Domeloeff L and Janunger KG. "The risk for gastric carcinoma after partial gastrectomy". The American Journal of Surgery 134 (1977): 581-584.

12. Helsingen $\mathrm{N}$ and Hillsted L. "Cancer development in the gastric stump after partial gastrectomy for ulcer". Annals of Surgery 143 (1956): 173-179.
13. Klause J. "Late prognosis after partial gastrectomy for ulcer. A follow-up study of 961 patients operated upon from 1905 to 1933". Acta Chirurgica Scandinavica 114 (1958): b341-354.

14. Offenhause GJA., et al. "Mortality caused by stomachcancer after remote partial gastrectomy for benign conditions:40 years of follow up of an Amsterdam cohort of 2633 post gastrectomy patients". Gut 29 (1988): 1588-1590.

15. Northfield TC and Hall C. "Carcinoma of the gastric stump. Risks and pathogenesis". Gut 31 (1990): 1217-1219.

16. Langhans P., et al. "Operation-sequel carcinoma of the stomach. Experimental studies of surgical techniques with or without resection". World Journal of Surgery 5 (1981): 595-605.

17. Wang L., et al. "Gastritis cystica profunda recurrence after surgical resection: 2-year follow-up:a case report". World Journal of Surgical Oncology 12 (2014): 133.

18. Littler ER and Gleibermann E. "Gastritis cystica polyposa (Gastric mucosal prolapse at gastrectomy site, with cystic and infiltrative epithelial hyperplasia)". Cancer 29 (1972): 205-209.

19. Ozenc AM., et al. "Gastritis cystica polyposa". Archives of Surgery 123 (1988): 372-373.

20. Wu MT., et al. "CT of gastritis cystica polyposa”. Abdominal Imaging 19 (1994): 8-10.

21. Park JS., et al. "Endoscopic treatment of gastritis cystica polyposa found in an unoperated stomach". Gastrointestinal Endoscopy 54 (2001): 101-103.

22. Tuncer K., et al. "Gastritis cystica polyposa found in an unoperated stomach: an unusual case treated by endoscopic polypectomy". Endoscopy 35 (2003): 882.

23. Li C., et al. "Gastritis cystica profunda: clinical and pathologic study of seven cases and review of literature". International Journal of Clinical and Experimental Pathology 14.2 (2021): 261-266.

24. XU XF., et al. "Gastritis cystica profunda in a previously unoperated stomach: a case report". World Journal of Gastroenterology 21.12 (2015): 3759-3762. 
25. Bechade D., et al. "Gastritis cystica profunda in a patient with no history of gastric surgery: a case report". Endoscopy 39 (2007): E80-E81.

26. Itte V., et al. "Massive gastrointestinal haemorrhage due to gastritis cystica profunda: a case report". Cases Journal 1.1 (2008): 85.

\section{Volume 4 Issue 7 July 2021}

(C) All rights are reserved by Seyed Reza Fatemi., et al. 